\title{
Editorial
}

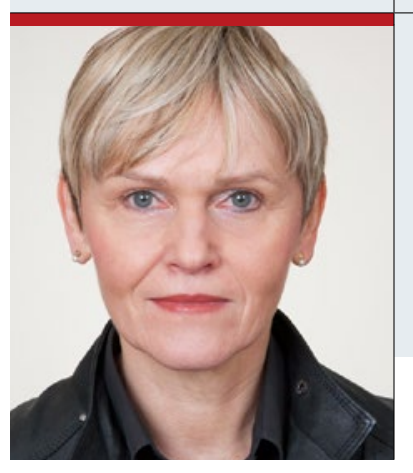

\section{Heike Ottow}

Leitende Redakteurin

heike.ottow@springer.com

facebook.com/springerpflege

\section{Kühler Kopf und heißer Sommer}

$\mathrm{B}$ estimmt kennen Sie das: Die Situation ist wieder einmal total stressig. Sie fühlen sich überfordert. Gerade Pflegende werden täglich mit den Nöten und den körperlichen Leiden pflegebedürftiger Menschen konfrontiert und erfahren häufig die Grenzen ihrer beruflichen oder persönlichen Interventionsmöglichkeiten. Aber es gibt Kollegen, die sich auch in solchen Momenten nicht unterkriegen lassen. Sie bewahren einen kühlen Kopf, wenn sich die Probleme häufen. Ist der Zeitdruck hoch, schaffen sie es, ihn auszugleichen. Resilienz heißt diese psychische Widerstandsfähigkeit. Die gute Nachricht ist, dass jeder Resilienz erlernen kann. Wie das funktioniert, beschreibt unser Autor Siegfried Huhn in einem Beitrag des PflegeKollegs ,Sich selbst pflegen“" (Seite 13). In diesem PflegeKolleg geht es auch um Achtsamkeit. Auch sie kann man trainieren. Viele Kliniken bieten deshalb Mitarbeitenden Kurse in Achtsamkeitsmeditationen. Gelehrt wird hier die Methode der MindfulnessBased-Stress-Reduction (MBSR). Die meist achtwöchigen MBSR-Kurse werden von den gesetzlichen Krankenkassen als Methode der gesundheitlichen
Prävention anerkannt und in der Regel bezuschusst (Seite 10).

Ich möchte Ihre Aufmerksamkeit besonders auf die HEILBERUFE-Serie „Digital“ lenken. In dieser und den folgenden Ausgaben stellen wir Ihnen die neue digitale Welt der Pflege vor. Dabei gehen wir Fragen nach wie: Welche Erfahrungen wurden in einer Einrichtung mit assistiven Technologien gesammelt? Wie bringt ein Netzwerk Anbieter innovativer Lösungen und Pflegefachkräfte zusammen?

Und, der Jahreszeit gemäß - schließlich hoffen wir auf einen heißen Sommer - beantworten wir die Frage: Wie viel Wasser braucht der Mensch? Bleibt mir, Ihnen im Namen der Redaktion einen schönen Sommer zu wünschen. Ihre
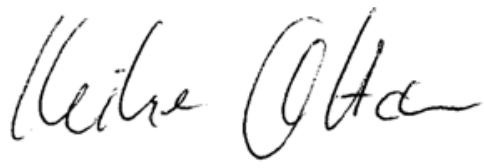

HEILBERUFE erscheint in diesem Monat mit einer Doppelnummer - die nächste Ausgabe erhalten Sie am 4. September.

\section{SPRINGER PFLEGE BEI FACEBOOK}

\section{Informieren, unterhalten, diskutieren}

Facebook erreicht alle. Das soziale Netzwerk ist längst keine Spielwiese mehr nur für Jugendliche. Auch viele Pflegende aller Altersgruppen schauen regelmäßig auf Facebook nach Neuigkeiten. Der offizielle Auftritt von Springer Pflege hat aktuell mehr als 15.000 Fans. Sie erhalten auf diesem Wege aktuelle Meldungen aus der Pflege, etwa zu politischen Entwicklungen und Veranstaltungen. Auch Neuigkeiten und Fotos von Kongressen finden hier ihren Weg zu allen Interessierten. Zudem hat auch Augenzwinkerndes aus dem Alltag auf Facebook seinen Platz. Und das Beste: Alle mit Interesse an der Pflege finden hier einen Ort, an dem sie über Themen diskutieren können, die sie bewegen.

www.facebook.com/SpringerPflege

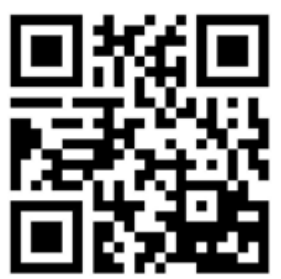

